\title{
The Effect of Closely-Coupled Interaction on Player Experience in Casual Games
}

\author{
Anastasiia Beznosyk, Peter Quax, Wim Lamotte, and Karin Coninx \\ Hasselt University - tUL - IBBT \\ Expertise Centre for Digital Media \\ Wetenschapspark 2 \\ 3590 Diepenbeek, Belgium
}

\begin{abstract}
This paper presents a study investigating cooperation between players in casual games. Although widely used in co-located or asynchronous settings, cooperative gameplay elements are not popular in networked synchronous casual games. In our study, we have analyzed different types of cooperation between players in casual games. Each of these is based on a certain cooperative game design pattern, and can be classified as either closely- or loosely-coupled. Six game patterns have been selected and an equal number of games developed, each targeting one pattern. By means of a user experiment we have investigated which cooperation types fit most of the criteria that define casual games. More specifically, we have focused on the applicability of close coupling between players. Based on the games used in the experiment, most patterns with closely-coupled interaction have shown an overall higher user evaluation than loosely-coupled, satisfying criteria of casual games. These results indicate that introducing close coupling in the casual games under consideration is a potential way to increase the player experience.
\end{abstract}

Keywords: casual games, cooperative game patterns, closely-coupled interaction, loosely-coupled interaction.

\section{Introduction and Related Work}

Over the last decade, casual games have become one of the most popular game type played over the Internet. These video games, targeted at a mass audience of casual gamers, attract because of their gameplay simplicity, a short play time and a minimum of required commitments to progress in a game [1. Additional popularity of casual games is caused by the rise of social networks (e.g. Facebook) and the availability of various game consoles (e.g. Wii) and mobile devices (e.g. smartphones, tablets) that allow competition and cooperation among friends 23]. Studies on digital games have shown that social characteristics of play settings have a strong impact on players' in-game experience [4.

Leaving competitive casual games out of the scope of this paper, our work focuses on cooperation in casual games. While intensively addressed in hardcore gaming (where gamers prefer to take significant time and develop their skill level, 
like in role-playing games), cooperation has not been widely investigated in the context of casual games. Here, most existing works focus on asynchronous interaction [5], where simultaneous play is not required. This type of collaboration is widely used for games on social networks (FarmVille1 or CityVille2). With the increased use of social games the game producers started to utilize this type of play [6], allowing players to play a game in sequence and break whenever is needed to "accommodate real life necessities". Casual games with synchronous collaboration appear to be mainly co-located. Players share the same screen and can naturally communicate and see each other. Games like Rock Band 3 and Mario Bros. Wit 4 are successful examples of synchronous cooperative casual games.

In most cases, games played over distance, which employ synchronous features, are limited to communication such as different forms of chats 7. Existing cooperative games that can be played remotely, are mainly based on loosely-coupled interaction (e.g. achieving a shared goal) without direct players' influence on each other. To our knowledge, closely-coupled collaboration, where one player's actions are directly influenced by the other(s), remains limited in existing casual games. One of the possible reasons for that can be a necessity to actively communicate and coordinate actions when tightly collaborating. Casual games are characterized by short game sessions oppositely to hardcore games, which take a substantial amount of time. Therefore, providing rich voice communication in casual games may become cumbersome for game developers or players might not want to be heard (e.g. playing during breaks at work).

We investigate the application of synchronous cooperation in remote casual games where no communication exists between players. By analyzing different types of cooperation (based on cooperative game patterns $[8]$ ), we aim to study the effect of closely-coupled collaboration on player experience in casual games through a comparison with loosely-coupled interaction.

\subsection{Cooperative Patterns}

Patterns in game design have been widely investigated 910 11. Game patterns are descriptions of reoccurring interaction, that depict how game components are used by players to affect various aspects of gameplay 11]. Since cooperation became an integral part of many multiplayer games and virtual communities, defining and analyzing effective cooperative patterns are the primarily goals in game design studies 812 .

The authors of [13] define collaboration patterns for knowledge sharing in Second Lif 5 based on the purpose of interaction (e.g. virtual meeting, design studio). Interaction patterns in massive multiplayer games (based on the

\footnotetext{
${ }^{1}$ WWW.facebook.com/farmville

2 ww. facebook.com/cityville

3 WWW.rockband.com

4 Www.mariobroswii.com

${ }^{5}$ WWW. secondlife.com
} 
example of Star War Galaxies 6 ) are investigated by Ducheneaut et al. in 14. Here the authors focus on the social aspects of interaction (verbal and non-verbal communication between players). Such cooperative patterns as turn-taking and enforced collaboration are studied by Goh et al. [15].

Studies presented in 81216 investigate cooperative patterns based on existing games. In [16], Zagal et al. explore cooperative patterns within traditional board games, and summarize observations that can be applied to computer games. Work presented by Rocha et al. [12 introduces a list of cooperative patterns based on the analysis of numerous commercial computer games available on the market. This list is considerably extended by El-Nasr et al. [ 8 ] by analyzing more recent games that support some form of collaboration between players. Here, the authors also investigate which pattern triggers which event (e.g. laughing, discussions, waiting for other players, etc.) during the shared gameplay.

Although cooperative patterns have been widely analyzed [16 128], these works always assume presence of communication between players or even an ability to see each other. Oppositely to the existing studies, our work focuses on collaboration in games where communication is not supported and players are remotely located. When considering interaction over distance, especially when no communication is allowed, additional challenges are present when trying to maintain the same level of player experience. Games based on the same cooperative patterns can result in an entirely different experience when considered in a non-collocated setup. Moreover, for our research a consistent game design is utilized to provide players with an equal perception of the game world while highlighting the impact of each interaction pattern. Most previous works are based on the existing games, where the same patterns are evaluated in different games that may influence player experience. Therefore, we find it crucial to investigate how interaction between players can be improved based on introducing different cooperative patterns in order to compensate a possible negative effect due to a lack of communication. We believe, knowing this may help game developers improve player interaction in cooperative multiplayer casual games.

\section{Experiment}

In order to analyze synchronous cooperation in remote casual games, an experiment was conducted. During the experiment pairs of participants had to evaluate six games, each based on one of the cooperative patterns. A selection of patterns (selected from the list presented in [8]) was made based on their popularity and the frequency of appearance in existing multiplayer games. Based on the coupling between players, we grouped the selected patterns either in closely- or loosely-coupled type of interaction. Our previous studies have shown a positive player reaction towards closely-coupled collaboration in comparison with looselycoupled when accomplishing game-like tasks in a 3D virtual environment [17. In our current work, we investigate whether closely-coupled interaction can be

\footnotetext{
6 WWW. soe.com
} 
beneficial for casual game design by providing players with a better experience than loose coupling. In order to decide upon the suitability of the cooperation type in the context of casual games, each game was analyzed based on five criteria: excitement, engagement, challenge, understandability and replay value (further referred as replayability).

\subsection{Participants}

We recruited thirty-six unpaid volunteers (thirty-one males and five females) to participate in the experiment. The average age of participants was 28 years old, from 21 to 38 years. Most of them had a computer science background and were recruited among university staff and students. According to self evaluation, the average player experience with any type of casual games was 3.19 on a scale from 1 (never played) to 5 (played a lot).

\section{$2.2 \quad$ Apparatus}

During the experiment two players were located in neighboring rooms separated by a hallway. Each player used a 15.4" laptop connected over a LAN. One of the laptops was a HP Compaq 8510p (Intel Core 2 Duo T8100, $2.1 \mathrm{GHz}, 3$ GB with ATI Mobile Radeon HD2600 graphic adapter) and the other was a Dell Latitude E6510 (Intel Core i3 M370, 2.4 GHz, 2 GB with NVIDIA NVS $3100 \mathrm{M})$. A separate external keyboard was attached to each laptop for a more comfortable input. No communication was possible between the two players. One observer was present in each room and sat beside the participant.

\subsection{Developed Cooperative Casual Games}

Six custom games were created for the experiment, each adopting one of the selected cooperative game patterns. Based on the coupling between players, we classified each game into one of two categories: closely- or loosely-coupled. If a game requires a lot of waiting or if the actions of one player directly affect the other player, it was categorized as the first type. The games that do not require tight collaboration between players and allow more independent performance were assigned to the second type.

For every game, a similar 3D virtual environment (Fig. II) was developed, which consists of several islands (a rectangular area, on which all game elements are located). Players are represented by alien-like avatars used from Unity 3D tutoria $\sqrt{7}$. To distinguish the two avatars in the virtual environment, one is colored in a light blue color, while the other avatar is brown. Players are able to navigate freely in the environment and are not forced to stay together. They have to collect different objects by running over them. Some of the objects are located on higher platforms not directly reachable by the players. Therefore, they have to

7 available online at http://unity3d.com/support/resources/tutorials/3d-platform-game 
use the jumping pads that help a player to jump higher to collect certain items. In order to get on a different island players need to jump across the abyss. If one of the players falls off the island the team loses one life. In all games players have five lives. After a fall, the player reappears at one of the respawn points. A group score is calculated and analyzed to measure the successfulness of the game completion. All closely-coupled games have equal conditions: players need to pick up 75 objects within 7 minutes. Loosely-coupled games are not designed equally and their play conditions are described further individually. The duration of loosely-coupled games is limited to 5 minutes. The game continues until one of the following conditions is met: (1) players collect all required objects; (2) players lose all their lives; (3) the time runs out.

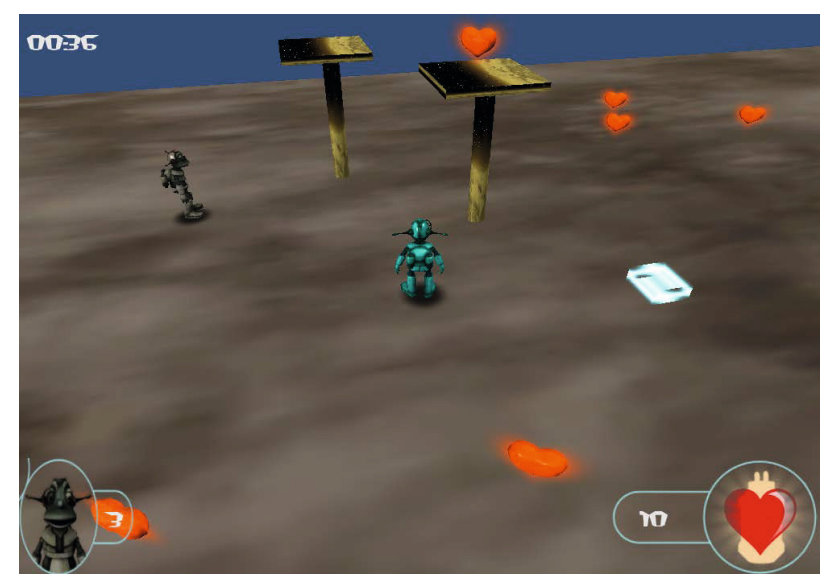

Fig. 1. An example of the game scene

A limited resources pattern is adopted for closely-coupled Game 1. Two players have to collect items, but are able to store a maximum of 10 items at the same time. Once both players reach the maximum amount of items, they can collect the following 10 objects. If one of the players collects 10 objects he/she has to wait for the other player and cannot pick up new items in the meantime.

A complementary pattern is used in closely-coupled Game 2, which implies that players have a different role to complement each others' activities within the game. During this game two roles are introduced. One player moves the jumping pad around the island while not being able to jump, and the other player uses it for jumping to reach objects located on higher platforms. There is only one jumping pad on each island. The roles are assigned randomly when players start the game.

An interaction with the same object pattern is followed in closely-coupled Game 3. In this game players have to move the jumping pad simultaneously. As soon as one of the players selects the jumping pad to move, the other player receives a message on his/her screen that the pad is selected and he/she is needed 
to help moving it. However, it does not indicate the location where the player has to be in order to help his/her teammate. When selected by two players, the jumping pad can be moved when both players walk in the same direction. Both players can use it for jumping. Similar to game 2, there is only one jumping pad on each island.

Loosely-coupled Game 4 utilizes a shared puzzles pattern. Here, the focus is to collect 10 special objects: each contains a heart with a letter on one side. Once all 10 special objects and therefore 10 letters are found, players need to use them to formulate a word containing all the letters, and put them in a designated window. The game succeeds when the word is entered correctly. Players do not see what words are entered by their partners while guessing. Once the correct solution is given by one player, the other one can also see it in his/her window.

An abilities that can be used on other players pattern is used in loosely-coupled

Game 5. In this game, players have to collect two types of objects: hearts and weapons. Each one is assigned to one player. They can see only one type which is randomly assigned on starting the game. Every time a player collects his/her 10 items, he/she gets the ability to see the partner's objects for about twenty seconds, and is able to collect them as well. The goal of the game is to collect 150 objects together, where every player has at least 50 objects of own type, and 15 objects of the partner's type.

A shared goals pattern is utilized in loosely-coupled Game 6. The collaboration is reduced to a shared goal of collecting 115 objects while acting independent from the partner.

\subsection{Procedure}

During the experiment, participant pairs completed consequentially six sessions, each corresponded to one of the casual games described earlier. In each game the player had to collaborate with his/her partner who was located in a different room. Like in many online games, players were coupled anonymously and, therefore, did not know who their partner was. Any form of communication (voice chat, text chat, pop-up messages, etc.) was avoided. Pop-up windows were used only in one game to support some basic level of awareness between two players. Before the actual experiment, a pilot test was performed to check the playability of every game.

Before the experiment, participants read a brief introduction and conducted a five-minute trial to familiarize themselves with the gaming environment and controls. In addition, written rules were given, in which both the goal and the way of interacting with the partner were explained. After each game, players were asked to evaluate the subjective perception of their experience. Based on the way they interact with the partner, they were asked to quantify the level of their excitement and engagement. Additionally, they evaluated how challenging and easy to understand each game was. Finally, they provided information regarding game replayability. For evaluation purposes, a visual analogue scale (VAS) was used. The participants marked on the $10 \mathrm{~cm}$ line the point that they felt represented their perception of the current state from not at all to very much. 
Also, the behavior of each player was observed and analyzed afterwards. It took approximately 60 minutes for each pair to complete the actual experiment.

\subsection{Design}

During the experiment, a within-subject design was used. The independent variable was the game type with six conditions. All participants, in pairs, had to complete six sessions testing every game type. The order of the conditions was counterbalanced using a balanced Latin square design. The dependent variables were excitement, engagement, challenge, understandability and replayability, so different components of a subjective evaluation of each game type. These were collected after each session through a questionnaire.

\subsection{Hypotheses}

To analyze six cooperative games from the perspective of their applicability for casual gaming, and more specifically to investigate the potential of closelycoupled games, we compare them based on five different criteria. While some of these criteria are chosen based on general requirements for computer games (excitement, engagement, challenge), the others represent characteristics more specific for casual games (understandability and replayability).

We state five hypotheses that apply to the games evaluated in our study. We want to see whether closely-coupled interaction, integrated in our games, can be of any advantage for casual gaming when compared to loose coupling. To check this assumption the following hypotheses are formulated with respect to the casual games criteria:

H1: each closely-coupled game provides higher excitement than each of the loosely-coupled games evaluated in the experiment;

H2: each closely-coupled game provides higher engagement than each of the loosely-coupled games evaluated in the experiment;

H3: each closely-coupled game provides more challenges than each of the looselycoupled games evaluated in the experiment without any negative impact on player excitement;

H4: each closely-coupled game does not provide additional difficulty to understand the interaction between players in the game when compared to each of the loosely-coupled games evaluated in the experiment;

H5: each closely-coupled game provides a higher level of replayability than each of the loosely-coupled games evaluated in the experiment.

\section{Results}

This section presents the results of our study. Firstly, analysis of the six games is reported based on five criteria: excitement, engagement, challenge, understandability and replayability. Secondly, we examine player experience based on the observations done during the experiment. 


\subsection{Subjective Player Evaluation}

During the experiment the six games were compared based on five criteria: excitement, engagement, challenge, understandability and replayability. Fig. [2] represents the averages and standard deviations of each criterion for every game based on the subjective player evaluation.

Although the games evaluated in the experiment share a lot of similar features like the graphics and in-game tasks (e.g. navigation, jumping, collecting objects), the interaction between players has been designed differently. Therefore, we check the hypotheses, presented in the previous section, separately for each closelycoupled pattern. By doing so we identify the closely-coupled patterns that improve casual gaming experience when compared with the loosely-coupled patterns. Table 1 summarizes the decision upon each hypothesis (accepted or rejected).

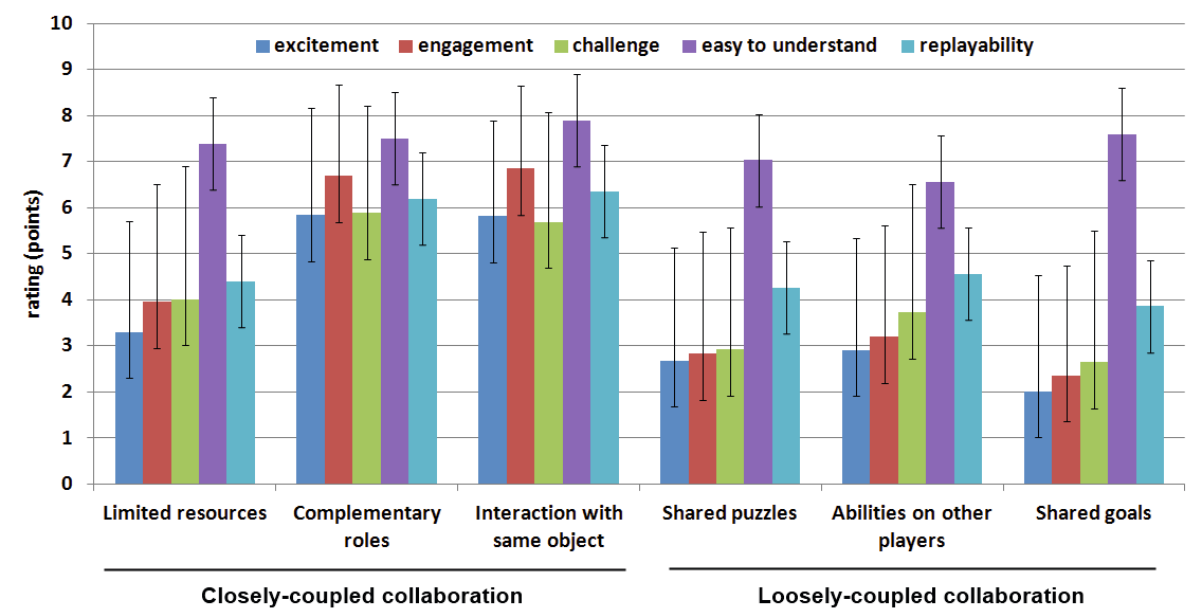

Fig. 2. Subjective player evaluation of the games

First of all, we analyzed player excitement in every game. From Fig. 2] we observe that closely-coupled games support a higher level of excitement. Repeated measures ANOVA with a Greenhouse-Geisser correction has shown a significant difference between the six games $(F(3.72,130.34)=28.72, p<0.01)$. A Bonferroni post-hoc test revealed that games 2 and 3 were found significantly more exciting $(p<0.01)$ than any of loosely-coupled games. Although game 1 showed an increase of player excitement when compared to the loosely-coupled games, the difference was not significant.

We have also discovered that not all closely-coupled games used in the experiment were equally exciting, as a significant difference existed among them. In particular, game 1 was found to be significantly less exiting $(p<0.01)$ than the other two closely-coupled games. One of the possible explanations for this could be the different nature of the in-game activities. Games 2 and 3 were the only ones where players had to move the jumping pad, which was not necessary in the other games. 
Table 1. Hypotheses check for the closely-coupled games evaluated in the experiment

\begin{tabular}{cccccc}
\hline $\begin{array}{c}\text { Pattern } \\
\text { type }\end{array}$ & H1 & H2 & H3 & H4 & H5 \\
& excitement & engagement & challenge & understandability & replayability \\
\hline \hline $\begin{array}{c}\text { Limited } \\
\text { resources }\end{array}$ & No & No & No & Yes & No \\
\hline $\begin{array}{c}\text { Complemen- } \\
\text { tary roles }\end{array}$ & Yes & Yes & Yes & Yes & Yes \\
\hline $\begin{array}{c}\text { Interaction } \\
\text { with same }\end{array}$ & Yes & Yes & Yes & Yes & Yes \\
object & & & & & \\
\hline
\end{tabular}

The second important characteristic is a high level of player engagement with a game. We asked players to evaluate how engaging they found cooperation in every game. Performed repeated measures ANOVA with a Greenhouse-Geisser correction has shown that not all games were similarly engaging $(F(3.75,131.22)$ $=36.54, p<0.01)$. Again we observed that games 2 and 3 obtained the highest points based on the players ratings. These two games were significantly more engaging than all loosely-coupled games $(p<0.01)$. Though being evaluated higher than the loosely-coupled games, game 1 did not significantly differ from them.

The next step was to analyze how challenging interaction between players in each game was. All games, except games 2 and 3, received a relatively low rating (on average for each game below 4 on the 0 to 10 scale). We found a significant difference among the six games $(F(5,175)=13.54, p<0.01)$. Both games 2 and 3 have shown to be more challenging than other games considered in the study $(p<0.01)$.

As additional challenges may decrease player enjoyment while playing a game, we investigated whether any negative influence existed in our case. This was particularly important for closely-coupled games, as this type of interaction was found to be more challenging. For each game, we checked the correlation between level of challenge and player excitement. Every of the six games has shown a significant positive correlation between these two parameters, indicating that in fact additional challenges, caused by the interaction between players in cooperative games, result in a higher enjoyment. This confirmed that more challenging closely-coupled games did not negatively impact player excitement.

The following criterion evaluated by players was understandability. As casual games imply easy understandable rules that do not require much experience, it is important that the way of interaction introduced in cooperative gaming does not decrease this understandability. All games have shown a high level of comprehension, without a significant difference across the six games $(F(4.11$, $143.87)=2.18, p>0.05)$. 
The last characteristic rated by players was replay value or replayability of the game. For casual games, it is a paramount characteristic that guarantees that people will be willing to play the same game again in the future without getting bored very quickly. Repeated measures ANOVA with a Greenhouse-Geisser correction has shown that the six games were not equal in this characteristic $(F(3.45,120.72)=9.97, p<0.01)$. Based on a Bonferroni post-hoc test, the games of the closely-coupled group have shown a higher player preference to replay the games again with a similar type of interaction between players $(p$ $<0.01)$. The highest values were obtained for games 2 and 3, showing a significantly higher level of replay value in comparison with all loosely-coupled games.

The obtained results have shown the difference among closely-coupled games, explained earlier in this section. As can be seen from Table 1 only one hypothesis was confirmed for game 1 . At the same time, games 2 and 3 were evaluated similarly based on all criteria due to the design resemblance between these two games. Every hypothesis was confirmed for games 2 and 3, indicating their high potential for casual game design.

\subsection{Observations of Players' Behaviour}

Besides the subjective player evaluation, we analyzed player experience based on the observations done during the experiment. We present these observations under three categories: players' emotions, performance and communication.For the latter, we consider different remarks that the participants gave about the communication component in cooperative games, as well as their attempts to communicate with the other player through the in-game actions.

Players' Emotions. Although several players were quite concentrated while playing, almost all of them showed a lot of positive emotions (smiling and laughing). In particular, this occurred when the player encountered his/her partner's avatar. Players were free to navigate in the environment, and therefore, could lose each other in the 3D world. This happened mainly during loosely-coupled games, where players did not stay together most of the time and rarely met each other. The positive reaction appeared also when the players were in each others' way, making the game a bit competitive by preventing the partner to pick up his/her objects directly. When being alone in the scene, players showed to be less emotional and were more focused on the task. They indicated that the looselycoupled games appeared to be more competitive than cooperative, with the goal of collecting more objects than the partner. Therefore, participants were focused on the task of getting more objects. Because most positive emotions were caused by (successful or not) interaction with another game character, loosely-coupled games resulted in less smiling or laughing than closely-coupled ones. One of the most 'emotional' games was game 3 , as it required almost constant interaction with a shared object and, thus, with the other player. Due to its nature, this game caused a lot of excitement among players. Positive emotions were not only caused by a successful play, but also, for example, while trying to move the same object in different directions. 
Performance. Observing player performance revealed the following aspects of cooperative interaction. In case of closely-coupled interaction, players tended to follow their partners all the time, in case help would be necessary during the game (mainly in games 2 and 3; but this also occasionally occurred in other games). If, for some reason, they lost each other in the environment, we observed players trying to find their partner as soon as possible, especially when they were waiting and could not proceed further without their help. As there was no communication, players tried to take into account all visual information in order to adjust their actions to partner's performance and help the partner. For example in game 2, once a player saw that his/her partner experienced difficulties in reaching objects on higher platforms, he/she immediately adjusted the position of the jumping pad. In loosely-coupled games players were free to decide whether or not they preferred to be alone or stay together with the other player. In contrast with this situation, closely-coupled games forced both players to be together or to wait for each other most of the time. We observed that a player acting extremely slow, made the experience of the other player less enjoyable.

Communication. During the experiment players were not allowed to communicate in any form. They learned from the game rules what they had to do, but could not discuss strategy or ask for help. Nevertheless, some of the players indicated that they were not affected too much by the absence of communication. In fact, they pointed out that the absence of communication made the game more challenging, and thus, more interesting and entertaining. They stated that information, they obtained from rules and what they observed on the screen, was sufficient for a successful gameplay. Based on the observations, we concluded that this was mainly typical for the evaluated closely-coupled games, as players were together most of the time and were aware of each others' actions. Here, it was not required to search for another player, making the need of communication less strong. In loosely-coupled games, players were not always sharing the same area of the virtual world and, therefore, they were not always aware about the partner's actions.

While observing participants, we noticed frequent occurrence of "communication with the screen". Players tried to explain their partners what they had to do, yet knowing that their messages could not be heard. Being not able to talk, players tried to find out ways in which they could assist their partners when it was obvious that he/she experienced difficulties. One of the solutions, we observed, was an attempt to 'communicate' an advice by moving his/her own avatar in front of the other player. By his/her own action, a 'better' player showed where the other one had to be for an easier accomplishment of the task.

Most games were played successfully under the given conditions of the remote setup without communication. However, half of the participants stated that the presence of communication would increase their performance. Although it was not proven by letting them play with communication, players pointed out that an ability to talk (either via text messages or voice) would make them more efficient. In particular, they mentioned an advantage of communication in order to divide 
areas for objects search. It was often the case that players lost a lot of time by going to the areas, where their partner had already harvested all objects. In general, participants expressed a strong wish for being able to talk with the other player even when it was not absolutely necessary for the game. For instance, if the strategy or in-game tasks were clear without actual communication, players still wanted to talk in order to make fun of each other and joke together.

\section{Conclusion}

In this paper we presented a study investigating synchronous cooperation in remote casual games where no communication existed between players. We analyzed six different types of cooperation (based on cooperative game patterns), each being either closely- or loosely-coupled. It has been shown that introducing closely-coupled interaction in casual games leads to a better player experience. We have observed that two of the three evaluated closely-coupled games introduced a higher level of player excitement, engagement and replayability without additional learning difficulties. In particular, games 2 (complementary roles pattern) and 3 (interaction with the same object pattern) satisfied all 5 criteria considered in the study, showing the advantage over the loosely-coupled games evaluated in the experiment.

Although we have seen player preference towards closely-coupled interaction in games, not all closely-coupled games were evaluated equally. Due to the possibility to design closely-coupled tasks differently, we realize the impact of game design on player evaluation. From the results, we observed that the design of games 2 and 3 differs more from the one of game 1 (limited resources pattern) than from each other.

With this study we have made the first attempt to evaluate cooperative game patterns in remote setup where any form of communication was not supported. Comparing results of our study with the existing works allows us to draw conclusions about an impact of the setup on the player experience 18. In particular, findings reported in 8 showed a higher contribution of several loosely-coupled patterns to the overall player experience (e.g. shared puzzles), while in our case close coupling was more preferred among players. We believe that such difference between remote and co-located setups is caused by the inability to talk.

Based on these findings, we can conclude that integration of closely-coupled interaction together with an appropriate game design has great potential for casual gaming. This triggers further research to confirm the obtained findings and generalize them to other games. By doing so it is possible define ways of making closely-coupled interaction more interesting for cooperative casual games among distributed players.

Acknowledgments. The research described in this paper is directly funded by Hasselt University through the BOF framework. The authors would like to thank Tom De Weyer for his assistance with the implementation and all participants who contributed to this research by taking part in the experiment. 


\section{References}

1. The Nielsen Company: Insights on casual games: analysis of casual games for the PC (2009), http://blog.nielsen.com/nielsenwire/wp-content/uploads/ 2009/09/GamerReport.pdf

2. Li, K.A., Counts, S.: Exploring social interactions and attributes of casual multiplayer mobile gaming. In: Proc. Mobility 2007, pp. 696-703. ACM (2007)

3. Mueller, F.'., Gibbs, M.R.: Evaluating a distributed physical leisure game for three players. In: Proc. OZCHI 2007, pp. 143-150. ACM (2007)

4. Gajadhar, B., Nap, H., de Kort, Y., IJsselsteijn, W.: Let's sweep some mines together: Social interaction \& competition in casual games. In: Proc. Fun and Games 2010, pp. 19-30 (2010)

5. Bogost, I.: Asynchronous multiplay: Futures for casual multiplayer experience. In: Proc. Other Players Conference 2004 (2004), http://www. bogost. com/downloads/ i. $\% 20$ bogost $\% 20-\% 20$ asynchronous $\% 20$ multiplay.pdf

6. Di Loreto, I., Gouaich, A.: Social casual games success is not so casual (2010), http://hal.archives-ouvertes.fr/lirmm-00486934

7. Ricchetti, M.: What makes social games social? (2002), http://www.gamasutra. com/view/feature/6735

8. El-Nasr, M.S., Aghabeigi, B., Milam, D., Erfani, M., Lameman, B., Maygoli, H., Mah, S.: Understanding and evaluating cooperative games. In: Proc. CHI 2010, pp. 253-262. ACM (2010)

9. McGee, K.: Patterns and computer game design innovation. In: Proc. IE 2007, RMIT University, pp. 16:1-16:8 (2007)

10. Kreimeier, B.: The case for game design patterns (2002), http://www.gamasutra. com/view/feature/4261

11. Björk, S., Holopainen, J.: Patterns in Game Design (Game Development Series). Charles River Media, Inc. (2004)

12. Rocha, J.B., Mascarenhas, S.P.R.: Game mechanics for cooperative games. In: ZDN Digital Game, pp. 73-80 (2008)

13. Schmeil, A., Eppler, M.J.: Knowledge sharing and collaborative learning in Second Life: a classification of virtual 3D group interaction scripts. Journal of Universal Computer Science 14, 665-677 (2009)

14. Ducheneaut, N., Moore, R.J.: The social side of gaming: a study of interaction patterns in a massively multiplayer online game. In: Proc. CSCW 2004, pp. 360369. ACM (2004)

15. Goh, W.B., Fitriani, Ting, L.G., Shou, W., Goh, C.F., Menon, M., Tan, J., Cohen, L.G.: Potential challenges in collaborative game design for inclusive settings (2011), http://www.dfki.de/educationchi2011/

16. Zagal, J.P., Rick, J., Hsi, I.: Collaborative games: lessons learned from board games. Simulation and Gaming 37(1), 24-40 (2006)

17. Beznosyk, A., Quax, P., Coninx, K., Lamotte, W.: The influence of cooperative game design patterns for remote play on player experience. In: Proc. APCHI 2012. ACM (2012)

18. Beznosyk, A., Quax, P., Coninx, K., Lamotte, W.: User enjoyment and performance in collaborative and cooperative games in shared $3 \mathrm{~d}$ virtual environments. In: Proc. GRAPP 2011, pp. 302-307 (2011) 